\title{
Anwendbarkeit der ISO 17100 auf intralinguales Übersetzen in Leichte und Einfache Sprache
}

\section{Applicability of the ISO 17100 to intralingual translation into Easy and Plain Language}

Silvana Deilen

Universidad de Mainz

sideilen@uni-mainz.de

Orcid: https://orcid.org/0000-0002-2933-9257 Orcid: https://orcid.org/0000-0002-3277-7717
Christiane Maaß

Universidad de Hildesheim maass.leichtesprache@gmail.com

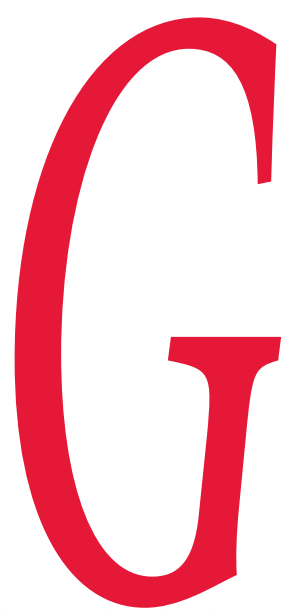

emäß dem novellierten Behindertengleichstellungsgesetz (BGG) sind „bauliche und sonstige Anlagen, Verkehrsmittel, technische Gebrauchsgegenstände, Systeme der Informationsverarbeitung, akustische und visuelle Informationsquellen und Kommunikationseinrichtungen sowie andere gestaltete Lebensbereiche [barrierefrei], wenn sie für Menschen mit Behinderungen in der allgemein üblichen Weise, ohne besondere Erschwernis und grundsätzlich ohne fremde Hilfe auffindbar, zugänglich und nutzbar sind“" ( $\$ 4$ BGG 2018, Hervorhebung durch die Autorinnen). Für den Bereich der Kommunikation wird diese Forderung nach Barrierefreiheit durch $₫ 11$ des BGG konkretisiert, in dem Träger öffentlicher Gewalt zur Bereitstellung von Informationen in Leichter Sprache verpflichtet werden. Das BGG führt somit aus, dass Menschen mit Behinderungen einen rechtlichen Bedarf an barrierefreien Informationen haben und dieser durch das Bereitstellen von Erläuterungen in Einfacher und Leichter Sprache erfüllt werden soll. Dies geschieht durch Übersetzen in Leichte Sprache und in Einfache Sprache, so dass sich das Thema im Bereich der intralingualen Übersetzung ansiedelt. Damit stellt sich die Frage, inwiefern Prozesse der Qualitätssicherung und -optimierung, die sich im Bereich der interlingualen Übersetzung herausgebildet und bewährt haben, auf die intralinguale Übersetzung in Leichte Sprache Anwendung finden können. Dieser Frage gehen wir im vorliegenden Beitrag nach. Dabei gehen wir insbesondere auf die Anwendbarkeit der Qualitätsnorm ISO 17100 auf das Übersetzen in Leichte Sprache ein. In Kapitel 2 diskutieren wir zunächst, was Leichte und Einfache Sprache sind, und zeigen anschließend in Kapitel 3 auf, inwiefern die aktuelle Textpraxis in den Bereich der Übersetzung fällt. Kapitel 4 ist dann den Instrumenten und Prozessen der Qualitätssicherung und -optimierung in der interlingualen Übersetzung und der Übertragbarkeit dieser Instrumente und Prozesse auf die Leichte Sprache gewidmet. Abschließend werden im fünften Kapitel Perspektiven für die Professionalisierung im Bereich der Barrierefreien Kommunikation diskutiert. 


\section{Zusammenfassung:}

Nach dem aktuellen Stand des Behindertengleichstellungsgesetzes (BGG, 2018) sollen Kommunikationen in Leichter und Einfacher Sprache zugänglich gemacht werden. Diese Tätigkeiten sind im Bereich der intralingualen Übersetzung angesiedelt, die ihrerseits Teil der kommunikativen Barrierefreiheit sind. Es stellt sich mithin die Frage, inwiefern Instrumente und Prozesse der Qualitätssicherung und -optimierung, wie sie sich für die interlinguale Übersetzung bewährt haben, auf die intralinguale Übersetzung in Leichte und Einfache Sprache anwenden lassen. Im vorliegenden Beitrag gehen wir dieser Frage insbesondere mit Blick auf die ISO 17100 nach, die den Standard für Übersetzungsdienstleister definiert und Übersetzungsqualität über die Prozessqualität definiert.

Schlüsselwörter: Leichte Sprache, Einfache Sprache; intralinguale Übersetzung, Übersetzungsqualität

\section{Abstract:}

According to the current status of the Act on Equal Opportunities for Persons with Disabilities (Behindertengleichstellungsgesetz, BGG, 2018), texts should be accessible in Easy and Plain Language. These activities are considered as intralingual translation, which in turn is part of communicative accessibility. Against this background, the question arises to what extent instruments and processes of quality assurance and optimization, which are established for interlingual translation, can be adapted to intralingual translation into Easy and Plain Language. In this article, we address this question in particular in view of the ISO 17100, which defines the standard procedures for translation service providers and translation quality through process quality.

Keywords: Easy Language, Plain Language, intralingual translation, translation quality

\section{Leichte und Einfache Sprache}

Leichte Sprache ist inzwischen in Politik und Gesetzgebung, Praxis und Wissenschaft fest etabliert. Die Großschreibung („Leichte Sprache”) verweist auf den Status als Eigenname und es liegen sowohl aus der Praxis (Netzwerk Leichte Sprache 2013, Inclusion Europe 2009) wie aus der Wissenschaft (Maaß 2015; Bredel/ Maaß 2016a, b) Handreichungen und Regelwerke vor. Für Einfache Sprache ist das nicht im selben Maße der Fall. Hier findet sich die Groß- neben der Kleinschreibung („Einfache Sprache” oder „einfache Sprache”), der Bekanntheits- wie auch der Regulierungsgrad dieser Varietät liegt insgesamt deutlich unter dem der Leichten Sprache.

Bei Leichter wie bei Einfacher Sprache handelt es sich um nähesprachlich geprägte verständlichkeitsoptimierte Reduktionsvarietäten des Deutschen (Bredel/Maßß 2016a: 32), die Personen mit Leseeinschränkungen den Zugang zu standard- und fachsprachlichen Informationen und somit kommunikative und gesellschaftliche Teilhabe ermöglichen. Ein Bedarf an verständlichkeitsoptimierter Kommunikation besteht bei unterschiedlichen Zielgruppen mit geringen Lesefähigkeiten mit und ohne Kommunikationsbehinderung. Die eingeschränkte Lesefähigkeit dieser Gruppen und infolgedessen der Bedarf an verständlichen, leicht wahrnehmbaren und barrierefreien Texten kann auf unterschiedliche Gründe zurückgehen: eine kognitive oder sensorische Beeinträchtigung, Lernschwierigkeiten, dementielle Erkrankungen, Aphasie, aber auch nicht behinderungsbedingte Formen des funktionalen Analphabetismus (vgl. Bredel/Maaß 2016a: 139ff.; eine Beschreibung der Barrieretypen in Abhängigkeit von den Behinderungsarten findet sich in Rink 2019 und 2020: 142ff.). Darüber hinaus sind auch Migrant(inn)en mit geringen Deutschkenntnissen, insbesondere zu Beginn des Zweitspracherwerbs, auf leicht verständliche Informationen angewiesen (vgl. Bredel/Maaß 2016a: 169ff.; Ahrens 2020). Das oberste Ziel der Textverständlichkeit wird in Leichter Sprache durch eine systematische Komplexitätsreduktion erreicht. Diese bezieht sich sowohl auf die einzelnen Ebenen des Sprachsystems als auch auf das bei den Rezipienten vorausgesetzte Sprach-, Welt- und Diskurswissen (vgl. Maaß 2015: 11f.; Rink 2020).

Zum Abbau von Kommunikationsbarrieren bedient sich die Leichte Sprache dabei einerseits „Strategien der Beschränkung (Reduktion)“ und andererseits „Strategien der Erweiterung (Addition)“ (Bredel/Maaß 2016b: 154f.). So wird durch die Beschränkung des lexikalischen und syntaktischen Inventars einerseits sichergestellt, dass ausschließlich leicht verständliche und dem/der Leser(in) bekannte sprachliche Strukturen verwendet 
werden. Andererseits wird das für das Textverständnis erforderliche und dem/der Leser(in) fehlende Sprach-, Welt- und Diskurswissen durch das Hinzufügen von Erläuterungen und Beispielen im Text angelegt (für eine ausführliche Beschreibung der Strategien der Reduktion und Addition s. Bredel/Maaß 2016a: 489ff.). Die Einfache Sprache ist weniger stark komplexitätsreduziert und erstreckt sich als Kontinuum zwischen Standard(und teilweise Fach-)sprache auf der einen Seite und der maximal komplexitätsreduzierten Leichten Sprache auf der anderen Seite. Bredel/Maaß (2016a: 526ff., 2016b: 186ff.) und Maaß (2020: 155ff.) beschreiben Einfache Sprache als strategische Anreicherung auf der Grundlage des Leichte-Sprache-Regelinventars.

Leichte Sprache richtet sich an den Bedarfen von Adressat(inn)en mit stärker ausgeprägten Kommunikationseinschränkungen aus und ist im engeren Sinne dem Feld der Barrierefreien Kommunikation zuzurechnen. Dabei ist der Begriff „barrierefrei” als relativ anzusehen: „Barrierefreiheit’ im wörtlichen Sinne [ist] eine Utopie“ (Maaß/Rink 2019: 21). Aufgrund der Heterogenität der Adressat(inn)en und der sich widersprechenden, unterschiedlichen kommunikativen Bedürfnisse ist eine generelle, zielgruppenübergreifende Barrierefreiheit im Bereich der Kommunikation nicht realisierbar. Durch die unterschiedlichen Bedarfe der Adressat(inn)en, die auch teilweise mit Mehrfachbehinderungen leben, führt das Streben nach Barrierefreiheit teilweise zu Zielkonflikten. Die Empfehlung von Leidner (2007), „dass bei jeder konkreten Maßnahme zur Herstellung von mehr Barrierefreiheit immer überlegt werden muss, ob nicht im selben Augenblick Barrieren für andere entstehen" (Leidner 2007: 32), kann auch auf den Bereich der Barrierefreien Kommunikation übertragen werden (Mälzer 2016:7). Folglich verweist der Terminus „Barrierefreiheit” nicht auf vollumfänglich barrierefreie, sondern vielmehr auf „barriereärmere[]' bzw. wahrnehmungs- und/oder verständlichkeitsoptimierte[] Kommunikate“ (Maaß/Rink 2019: 21).

\section{Leichte und Einfache Sprache als intralinguale Übersetzung}

Nach dem traditionellen Übersetzungsbegriff, der translation proper im Sinne von Jakobson (1959) bei der interlingualen Übersetzung verortet, fällt das Herstellen von Texten in Leichter und Einfacher Sprache auf der Grundlage standard- oder fachsprachlicher Ausgangstexte nicht in den Bereich der Übersetzung. Akteurs- und handlungsfeldbezogene Ansätze wie derjenige von Risku (1998 und öfter), die ihren Übersetzungsbegriff an den tatsächlichen Tätigkeiten der Übersetzer ausrichten, öffnen dagegen den Blick für andere Formen der Textarbeit, die dann ebenfalls als Übersetzung beschrieben werden kann: intersemiotische Formen der Übersetzung wie Audiodeskription oder Untertitelung für Hörgeschädigte und intralinguale Formen wie Übersetzen in Leichte und Einfache Sprache. Diese Formen sind sämtlich im Feld der Barrierefreien Kommunikation angesiedelt, das durch die Inklusionsbemühungen im Gefolge der UN-Behindertenrechtskonvention international erheblichen Aufschwung genommen und Märkte etabliert hat.

Übersetzen in Leichte und Einfache Sprache wäre damit, wiederum im Sinne Jakobsons (1959), als intralinguale Übersetzung zwischen unterschiedlichen Sprachvarietäten einer Einzelsprache zu definieren (Bredel/Maaß 2016a: 182ff.; Maaß 2019; Hansen-Schirra et al. 2020a). Dies deckt sich mit dem intuitiven Übersetzungsbegriff der Praxis: So wird von Seiten der Politik und Rechtsetzung (s. z. B. den Entwurf des novellierten BGG 2015: $4,40)$ von „Übersetzen in Leichte Sprache” gesprochen. In dieser Linie stehen auch die „Übersetzungsbüros” für Leichte Sprache der Lebenshilfe, die in den vergangenen Jahren mit Unterstützung der Aktion Mensch in zahlreichen deutschen Städten an den Start gegangen sind.

Die gesetzliche Forderung nach Barrierefreier Kommunikation hat dazu geführt, dass sich der noch junge Markt für barrierefreie Kommunikationsangebote in kurzer Zeit sehr vergrößert hat. Das stark anwachsende Volumen an Leichte-Sprache-Texten wird dabei in der derzeitigen Praxis nicht ausschließlich von professionell ausgebildeten Übersetzer(inne)n bewältigt, sondern in vielen Fällen werden die Aufträge an Büros für Leichte Sprache der Lebenshilfe oder anderer sozialer Organisationen vergeben. Diese stehen, im Unterschied zu vielen Übersetzungsbüros, durch die angeschlossenen Werkstätten im direkten Kontakt zu Vertreter(inne)n der primären Zielgruppe, verfügen jedoch häufig nicht über professionell ausgebildete Übersetzer(innen), sodass die Texte häufig von Akteur(inn)en ohne übersetzungswissenschaftlichen Hintergrund übersetzt werden. Dieser „teilweise ungeregelt[e] und intuitiv[e] [Markt]“(Maaß/Rink 2019: 17) hat zur Folge, dass das derzeitige Angebot 
an barrierefreien Kommunikaten durch eine äußerst heterogene Qualität gekennzeichnet ist (vgl. ebd.: 17f.).

Vor dem Hintergrund, dass die Berufsbezeichnung „Übersetzer” in Deutschland nicht geschützt ist, stellt die durchwachsene Textqualität im Bereich des inter- wie intralingualen Übersetzens eine bekannte Problematik dar. In der interlingualen Übersetzung wurde die Qualitätsproblematik in der Forschung intensiv diskursiviert (vgl. z. B. Holz-Mänttäri 1984) und in Folge in der Praxis über berufsverbandliche Strukturen und nationale wie internationale Standardisierungsbemühungen angegangen. Inzwischen steht zur Standardisierung der Qualitätssicherung in der interlingualen Übersetzung die internationale Qualitätsnorm DIN EN ISO 17100 zur Verfügung, in der Anforderungen an qualitativ hochwertige Übersetzungsdienstleistungen festgelegt sind. Eine Zertifizierung nach ISO 17100 hat sich auf dem Translationsmarkt als Kennzeichen dafür etabliert, dass sich die am Übersetzungsprozess beteiligten Akteur(inn)e(n) nachweislich dazu verpflichtet haben, höchsten Qualitätsstandards Rechnung zu tragen. Mit der Konformitätserklärung zu dieser Norm weisen die Übersetzungsdienstleister(innen) nach, dass sowohl die internen Prozesse als auch die Qualifizierung der beteiligten Akteur(inn)e(n) als qualitativ hochwertig eingestuft werden können. Das Produkt, das in solchen qualitätvollen Prozessen entsteht, entspricht nach DIN EN ISO 17100 dann ebenfalls den etablierten Qualitätsstandards (vgl. Bredel/Maaß 2016b: 70).

Obgleich eine „Orientierung an der prozessorientierten ISO-17100 Norm [...] [in der Leichte-SpracheÜbersetzung] noch nicht die übliche Praxis [ist]“(Bredel/Maaß2016b:72), kann eine Anlehnung an standardisierte Prozessabläufe und Qualitätsmerkmale auch beim Erstellen von barrierefreien Texten erheblich zur Verbesserung der instabilen Textqualität beitragen. Vor dem Hintergrund der Standardisierungsbestrebungen soll in dem vorliegenden Beitrag somit geprüft werden, inwiefern die in der DIN EN ISO 17100 genannten Anforderungen an Übersetzungsdienstleistungen auch auf den Bereich der Barrierefreien Kommunikation angewandt werden können bzw. für diesen modifiziert und ergänzt werden müssen. Dem wendet sich der vorliegende Beitrag in Folge zu.

\section{4. Übertragung der ISO 17100 auf intralinguales Übersetzen in Leichte und Einfache Sprache}

\subsection{Produktionsprozesse}

Ebenso wie für interlinguale Übersetzungsprojekte ist es auch für intralinguale Übersetzungsprojekte unabdingbar, dass diese von einem/einer Projektmanager(in) koordiniert werden. Der/die Projektmanager(in) ist u. a. dafür verantwortlich, kompetente Übersetzer(innen) und Revisor(inn)en auszuwählen, mit dem Kunden/ der Kundin zu kommunizieren und das Feedback einzuholen.

Auch beim Erstellen von Leichte-Sprache-Texten ist es die Aufgabe des Übersetzers/der Übersetzerin, „in Übereinstimmung mit dem Zweck des Übersetzungsprojektes, einschließlich der sprachlichen Konventionen der Zielsprache und der betreffenden Projektspezifikationen, [zu] übersetzen“" (DIN EN ISO 17100 2015: 16). Die in der ISO 17100 aufgeführten Unterpunkte können dabei jedoch nicht ausnahmslos auf den Übersetzungsprozess übertragen werden.

Angesichts der geringen Wortschatzkenntnisse der Rezipient(inn)en (Bredel/Maaß 2016a: 117ff.) und der daraus resultierenden erforderlichen konsistenten Terminologieverwendung (Maßß 2015: 34ff.) ist es für eine barrierefreie Übersetzung gemäß ISO 17100 von besonderer Relevanz, dass der/die Übersetzer(in) die vorher festgelegte ,spezielle[] Sachgebiets- und Kundenterminologie“ (DIN EN ISO 17100 2015: 16) einhält und sich der Notwendigkeit der terminologischen Konsistenz bewusst ist, was u. a. bedeutet, dass Synonyme oder sprachliche Varietät zu vermeiden sind. Auch die in der Norm festgelegten Forderungen nach semantischer Korrektheit sowie angemessener Syntax und Orthografie haben in Leichter Sprache einen hohen Stellenwert, denn gemäß des in Leichter Sprache geltenden Grundsatzes „falsches Deutsch vermeiden“ (Maaß 2015: 82ff.; zu durch inkorrekte Orthografie ausgelöste Stigmatisierungsprozesse s. Maaß 2020: 105ff.) sollten die komplexitätsreduzierenden Strategien nicht zu Lasten der grammatikalischen oder orthografischen Korrektheit gehen. Bei der Forderung nach angemessener Syntax, Orthografie, Zeichensetzung und diakritischen Zeichen ist allerdings zu beachten, dass neben dem Kriterium der Korrektheit zugleich auch die in Leichter Sprache herrschenden Restriktionen berücksichtigt werden müssen, die dazu führen, dass das grammatische Inventar der Leichten Sprache erheblich 
begrenzt ist (vgl. Bredel/Maaß 2016a). Aufgabe des Übersetzers/der Übersetzerin ist es somit, anhand von wenigen grammatischen Ausdrucksmöglichkeiten und erlaubten Satzkonstruktionen die im Ausgangstext enthaltenen, komplex formulierten Informationen präzise, verständlich und dennoch grammatikalisch und semantisch korrekt zu übersetzen.

Ebenso kann auch die in der Norm geforderte lexikalische Kohäsion und Phraseologie nicht uneingeschränkt auf die Leichte-Sprache-Übersetzung übertragen werden. Obgleich Kohäsion, also der syntaktisch-grammatische Zusammenhang des Textes, auch in Leichter Sprache zu den essenziellen Texteigenschaften zählt, wird das Herstellen von Kohäsion in Leichte-Sprache-Texten dadurch erschwert, dass viele kohäsionsstiftende Mittel aufgrund der Reduktion des lexikalischen und grammatischen Inventars nicht zur Verfügung stehen, wie $\mathrm{z}$. B. ein Großteil der verbindenden satzinternen Konjunktionen (Bredel/Maaß 2016a: 391ff.), die pronominale Wiederaufnahme (Maaß 2015: 68ff.) oder der Gebrauch von Synonymen und Hyperonymen. Zur Herstellung von Kohäsion wird in Leichte-Sprache-Texten stattdessen überaus häufig auf die Rekurrenz zurückgegriffen. Ebenso sind viele feste Wortverbindungen und konventionelle Phrasen wie komplexe Nominal- oder Präpositionalphrasen aufgrund inhärenter lexikalischer und/oder grammatischer Komplexität nicht lizenziert (vgl. Bredel/Maaß 2016a; Wolfer 2017; Rink 2020). Die lexikalischen und syntaktischen Restriktionen führen wiederum dazu, dass auch konventionelle Formulierungen und Phrasen, die auf eine bestimmte Textsorte und somit auf die Textfunktion verweisen und zugleich Orientierung liefern („Im Namen des Volkes”), in Leichter Sprache nur sehr begrenzt verfügbar sind. Während in der Standardsprache die Bandbreite der lexikalischen, syntaktischen und phraseologischen Mittel in den einzelnen Textsorten anhand von Textsortenkonventionen selektiv ausgeschöpft wird, führt das in Leichter Sprache stark begrenzte lexikalische und grammatische Repertoire zu einer morphologischen, lexikalischen und syntaktischen Homogenisierung der Texte (Bredel/Maaß 2016a; Rink 2020). Da diese Homogenisierung der Texte wiederum zur Folge hat, dass auch die Textfunktion in LeichteSprache-Texten nur bedingt realisiert und teilweise nur schwer erkannt werden kann, kommt dem Übersetzer/ der Übersetzerin, im Unterschied zur interlingualen Übersetzung, die Aufgabe zu, die Textfunktion explizit zu versprachlichen (Rink 2020; Maßß 2020). Der/Die Übersetzer(in) muss sich hierbei zudem darüber bewusst sein, dass es, im Unterschied zur interlingualen Übersetzung, oftmals nicht möglich ist, funktionskonstant zu übersetzen und die Übersetzung des Ausgangstextes somit häufig mit einem Funktionswandel einhergeht (Maaß et al. 2014). So kommt z. B. Rechtstexten eine rechtliche Absicherungsfunktion zu; die Leichte-SpracheVersion erfüllt diese Funktion hingegen nicht. Grund hierfür ist, dass ein Text nur dann rechtsverbindlich ist, wenn er bestimmte Standards erfüllt und Formulierungen enthält, diese jedoch angesichts ihrer Komplexität in der Leichte-Sprache-Übersetzung nicht übernommen werden können (vgl. Maaß 2015: 141). Das Kriterium der lexikalischen Kohäsion und Phraseologie gilt es somit zwar auch bei der Leichte-Sprache-Übersetzung zu berücksichtigen, der Zusatz „gemäß den sprachlichen Konventionen der Zielsprache” ist hierfür jedoch unentbehrlich.

Ein zentraler Unterschied zur interlingualen Übersetzung besteht in den bei der Wortauswahl zu beachtenden Kriterien. Während die Terminologieauswahl beim interlingualen Übersetzen häufig bereits durch Einträge in Terminologiedatenbanken festgelegt ist, ist es bei der Leichte-Sprache-Übersetzung die Aufgabe des Übersetzers/ der Übersetzerin, unter Berücksichtigung der Prototypentheorie und den damit einhergehenden Kriterien zu ermitteln, welches Wort die meisten der hoch priorisierten Kriterien erfüllt und somit für die Zieltextleser(innen) am verständlichsten ist (vgl. Bredel/Maaß 2016a: 347). Ebenso wie bei der interlingualen Übersetzung kann der Übersetzer/die Übersetzerin auch bei der Leichte-Sprache-Übersetzung von CAT-Tools (computer-aided translation) profitieren (Maaß et al. 2014), wobei zu beachten ist, dass die bestehenden CAT-Tools bislang nur eingeschränkt verwendet werden können. Im Unterschied zur Nutzung von CAT-Tools in der interlingualen Übersetzung müssen bei der intralingualen, barrierefreien Terminologiearbeit insbesondere beim Anlegen und Nutzen von Terminologieeinträgen einige Besonderheiten beachtet werden. So bedarf es bspw. Eingabefelder für Paraphrasen oder Erklärungen sowie Möglichkeiten zur intersemiotischen Übersetzung (zu den Möglichkeiten und Grenzen der Einbindung von CAT-Tools in Leichter Sprache s. Hansen-Schirra et al. 2020b). Dies impliziert zugleich, dass Leichte-Sprache-Übersetzer(innen) im Umgang mit multikodaler und multimodaler Übersetzung geschult und in der Lage sein müssen, den Zieltext je nach Bedürfnissen der Zieltextrezipient(inn)en multikodal 
und multimodal aufzubereiten.

Ebenso wie in der interlingualen Übersetzung ist es auch beim intralingualen barrierefreien Übersetzen von zentraler Bedeutung, dass der/die Übersetzer(in) die „eigenen und/oder kundenseitigen Stilrichtlinien (einschließlich Sachgebiet, Sprachregister und Sprachvarietät)“ (DIN EN ISO 17100 2015: 16) einhält. Hierbei ist zu beachten, dass der Bereich der Barrierefreien Kommunikation aus einem Kontinuum unterschiedlich stark komplexitätsreduzierter Varietäten besteht (Leichte Sprache, Leichte Sprache Plus, Einfache Sprache, Bürgernahe Sprache, vgl. Maaß 2020) und es je nach Reduktionsvarietät unterschiedliche Handlungsempfehlungen und Richtlinien zur Komplexitätsreduktion bzw. zur sprachlichen Anreicherung der Texte gibt. Im Gegensatz zur interlingualen Übersetzung gibt es allerdings keine fest vorgeschriebenen amtlichen Regeln für Orthografie und Grammatik der Zielsprache, sondern insbesondere der Bereich der Leichten Sprache zeichnet sich durch eine Reihe von konkurrierenden Regelwerken aus (für einen Überblick s. Maßß 2015: 26ff.; Maaß 2020: 69ff.). Folglich ist es zu Beginn des intralingualen Übersetzungsprozesses erforderlich, dass der Kunde/die Kundin eine Orientierung an einem konkreten Regelwerk vorgibt, an die sich der/die Übersetzer(in) ausnahmslos zu halten hat. Die obligatorische Einhaltung der kundenseitigen Stilrichtlinien kann allerdings dazu führen, dass der/ die Übersetzer(in) gegen den genannten Grundsatz „falsches Deutsch vermeiden“ (Maaß 2015: 82ff.) verstoßen muss, da in einigen Regelwerken bspw. postuliert wird, dass die Textverständlichkeit vor der Korrektheit der Texte Vorrang hat und somit orthografisch falsche Bindestrichkonstruktionen unter Umständen von dem Kunden/der Kundin gewünscht sein können.

Auch dem Unterpunkt der Formatierung kommt bei Leichte-Sprache-Übersetzungen eine zentrale Rolle zu. Im Unterschied zur interlingualen Übersetzung ist die Formatierung jedoch angesichts der unterschiedlichen Barrieren der Rezipient(inn)en weitgehend regelgeleitet und weicht grundsätzlich von der Formatierung des Ausgangstextes ab.

Darüber hinaus wird in der Norm vorgeschrieben, dass die Übersetzung unter Berücksichtigung der „Zielgruppe und Zweck des zielsprachlichen Inhalts“ (DIN EN ISO 17100 2015: 16) anzufertigen ist. Das Kriterium der Zielgruppenorientierung und der Funktionsgerechtigkeit kann auch beim Übersetzen in Leichte Sprache als zentrales Merkmal der Zieltextqualität betrachtet werden. So ist es die Aufgabe des Übersetzers/ der Übersetzerin, die Barrieren und Bedarfe der Rezipient(inn)en zu identifizieren und die im Ausgangstext enthaltenden Barrieren unter Anwendung der jeweils angemessenen Übersetzungsstrategien zu überwinden. Angesichts des geringen common grounds (Clark 1996; Rink 2020: 175ff.) zwischen dem Produzenten des Ausgangstextes und den Empfänger(inne)n des Zieltextes ist es dabei zugleich die Aufgabe des Übersetzers/ der Übersetzerin, das für das Textverständnis erforderliche Sprach-, Welt-, Erfahrungs- und Diskurswissen zu präsupponieren und, sofern es bei den Adressat(inn)en nicht vorausgesetzt werden kann, im Zieltext anzulegen. Da der/die Übersetzer(in) somit Wissenslücken antizipiert und den Zieltext an die Wissensvoraussetzungen der Rezipient(inn)en anpasst, fungiert er/sie als „Kenner(in) der Zielgruppe und Experte/Expertin für ihre kommunikativen Bedürfnisse“ (Bredel/Maaß 2016a: 208). Von den unterschiedlichen Paradigmen und Ansätzen, die die Geschichte des interlingualen Übersetzens geprägt haben (für einen Überblick s. Siever 2010), ist für das intralinguale Übersetzen in Leichte Sprache somit das handlungsorientierte Paradigma am bedeutendsten. Gemäß dem handlungsorientierten Paradigma erstellt der/die Übersetzer(in) als handelnde(r) Experte/Expertin funktionale Zieltexte, die an die Zielsituation und die intendierte Adressatenschaft angepasst sind. Dieser in Leichter Sprache dominierende Ansatz des funktionsgerechten, skopos- und handlungsorientierten Übersetzens steht somit, aus theoretischer Perspektive, in Einklang mit den in der Übersetzungswissenschaft führenden handlungstheoretischen Ansätzen, wie z. B. der Skopostheorie von Reiß/Vermeer (1984), der Theorie des translatorischen Handelns von Holz-Mänttäri (1984) oder der Theorie des funktionalen Übersetzens von Nord (1993) (s. dazu Maaß 2019). Ebenso wie in der interlingualen Übersetzung ist die Funktionsgerechtigkeit der Leichte-Sprache-Übersetzung erreicht, wenn das Translat in der Zielsituation den angestrebten oder geforderten Zweck erfüllt. Es geht folglich nicht um die Äquivalenz, sondern um die Adäquatheit des Zieltextes, d. h. das Ziel der Übersetzung ist nicht die Übereinstimmung mit dem Ausgangstext, sondern die Übereinstimmung mit dem Zweck und somit das Abbauen von Barrieren. Diese Adäquatheit des Zieltextes gilt es bei der intralingualen Übersetzung insbesondere auch bei dem „Dilemma“ (Maaß 2015: 130) der Informationsauswahl 
zu berücksichtigen. Eine Übernahme von allen Informationen ist in der Regel nicht möglich, da dies aufgrund der additiven Verfahren dazu führen würde, dass der Zieltext um ein Vielfaches länger wäre als der Ausgangstext, was angesichts der geringen Aufmerksamkeitsspanne der Leser(innen) im Widerspruch zu der geforderten Barrierefreiheit steht (s. Rink 2020 zu verschiedenen Übersetzungsszenarien). Folglich gilt es zu Beginn des Übersetzungsprozesses, in Abstimmung mit dem Kunden/der Kundin und unter Berücksichtigung der Textfunktion und der situativen Merkmale (Zielgruppe, Medium, Anlass etc.) die zentralen Aussagen und Aussageabsichten des Textes festzulegen. Die Auswahl der Informationen muss dabei gut motiviert sein und darf nicht auf Basis von subjektiven Präferenzen des Übersetzers/der Übersetzerin erfolgen.

In Hinblick auf den in der ISO 17100 aufgeführten Teilprozess der Übersetzung ergibt sich für den/die Übersetzer(in) somit, ebenso wie bei der interlingualen Experten-Laien-Kommunikation, die Herausforderung, die Komplexität der fachsprachlichen Informationen des Ausgangstextes zu reduzieren und das für das Textverständnis erforderliche Wissen in sprachlich und inhaltlich komplexitätsreduzierter Form im Zieltext $\mathrm{zu}$ vermitteln. Im Unterschied zur Experten-Laien-Kommunikation bezieht sich das Wissensgefälle in der Leichte-Sprache-Übersetzung jedoch nicht nur auf das Fach- und Fachsprachenwissen, sondern auch auf die schriftsprachliche Kompetenz und teilweise auch auf die Sprachfähigkeit, die visuelle oder auditive Wahrnehmungsfähigkeit und die kognitiven Fähigkeiten. Im Unterschied zur interlingualen Übersetzung ist der/die Übersetzer(in) somit nicht nur Kenner(in) der Sprache und des Fachgebietes, sondern darüber hinaus Kenner(in) der Text- und Rezipientenbarrieren sowie Kenner(in) der Rezipientenfähigkeiten und Bedürfnisse. Diese Anforderung an den/die Übersetzer(in) wird jedoch durch die Heterogenität und die unterschiedlichen kommunikativen Bedürfnisse der Zielgruppen erheblich erschwert (für einen Überblick über die jeweiligen Kommunikationsbarrieren der einzelnen Zielgruppen s. Rink 2020).

Während die in der Norm vorgeschriebene Kontrolle des zielsprachlichen Inhalts durch den/die Übersetzer(in) uneingeschränkt auf das Übersetzen in Leichte Sprache übertragen werden kann, lassen sich in der darauf folgenden obligatorischen Revision wesentliche Unterschiede zwischen der derzeitigen interlingualen und intralingualen Übersetzungspraxis konstatieren. So wird in der DIN EN ISO 17100 festgelegt, dass „,[d]er Revisor, der eine andere Person als der Übersetzer sein muss, [...] die unter 3.1.5 genannten Kompetenzen in der Ausgangsund in der Zielsprache aufweisen [muss]“ (DIN EN ISO 17100 2015: 17). Der/Die Revisor(in) muss dazu fähig sein „den zielsprachlichen Inhalt im Vergleich mit dem Original auf jegliche Fehler und andere Probleme sowie auf ihre Zweckentsprechung [zu] prüfen“ (ebd.). Da die Revision gemäß der ISO 17100 ein zentraler Bestandteil der Qualitätssicherung für Übersetzungsdienstleister(innen) ist, sollte somit auch bei der intralingualen Übersetzungsdienstleistung sichergestellt werden, dass die Übersetzung einen Revisionsprozess durchläuft. Das Thema Qualitätssicherung hat in der derzeitigen Leichte-Sprache-Praxis einen hohen Stellenwert; sie wird jedoch zugleich kontrovers diskutiert. Zwar besteht sowohl in der Praxis als auch in der Wissenschaft Konsens darüber, dass die Revision ebenso wie in dem interlingualen Übersetzungsprozess von einem/einer dritten Akteur(in) durchzuführen ist, jedoch gibt es für die Auswahl des Revisors/der Revisorin unterschiedliche Ansätze. Vor dem Hintergrund, dass die Leichte Sprache ursprünglich für Menschen mit kognitiven Beeinträchtigungen entwickelt wurde, wird in der Praxis dafür plädiert, dass ein Text nur dann als Leichte-Sprache-Text ausgewiesen werden darf, wenn er zuvor eine Revision durch eine Prüfgruppe durchlaufen hat (vgl. Netzwerk Leichte Sprache 2013: 35). Hierbei wird der Zieltext von Vertreter(inne)n der primären Zielgruppe auf Verständlichkeit überprüft. Im Gegensatz zur interlingualen Übersetzung wird der Ausgangstext bei dieser Form der Revision gänzlich außer Acht gelassen. Erst nachdem die Korrekturen zur Zufriedenheit der Prüfgruppe eingearbeitet wurden, darf der Zieltext an den Kunden/die Kundin ausgeliefert werden. Die derzeit praktizierte Zielgruppenkorrektur kann auf Basis der ISO 17100 allerdings aufgrund des fehlenden Vergleichs von Ausgangs- und Zieltext nicht als Revision bezeichnet werden. Dies ist darauf zurückzuführen, dass sich die Prüfer(innen) gerade dadurch auszeichnen, dass sie den Ausgangstext aufgrund ihrer geringen Lesekompetenzen nicht verstehen und folglich - selbst wenn sie den Ausgangstext zur Verfügung hätten - auch nicht beurteilen könnten, ob der Zieltext den Inhalt des Ausgangstextes korrekt wiedergibt. Auch die Tatsache, dass die Prüfer(innen) häufig willkürlich und nicht anhand von vorher festgelegten Kriterien ausgewählt werden, steht im Widerspruch zu den in der Qualitätsnorm festgelegten Standards. Problematisch an dem derzeitigen Revisionsverfahren ist zudem, dass die Textrezeption 
„häufig schwerpunktmäßig auditiv“ (Bredel/Maaß 2016a: 177) erfolgt. Dass die Texte den Prüfer(inne)n vorgelesen werden, widerspricht jedoch dem Prinzip der Barrierefreiheit und dem Ziel, die Informationen so aufzubereiten, dass sie "grundsätzlich ohne fremde Hilfe [...] zugänglich und nutzbar sind“ (\$ 4 BGG 2018). Die Prüfsituation stellt somit keine authentische Rezeptionssituation dar, denn beim Vorlesen werden dem Text zum einen durch Stimmmerkmale wie Tempo, Pausen und Intonation paraverbale Informationen hinzugefügt (Bredel/Maaß 2016a: 177). Zum anderen wird der Text oftmals satzweise vorgelesen, was dazu führt, dass die Verständlichkeit auf Textebene nicht beurteilt werden kann. Ob der Text seine Funktion in der Zielsituation erfüllt, kann mit einer auditiven Rezeption somit nicht hinreichend geprüft werden. Für eine zielführende Textprüfung ist es folglich erforderlich, dass die Rezeptionsbedingungen der Prüfsituation weitgehend denen der Zielsituation entsprechen. Aufgrund der genannten Schwachstellen der Zielgruppenprüfung werden jedoch darüber hinaus noch alternative Revisionsverfahren eingesetzt, bei denen der Text anhand des in der ISO 17100 vorgeschriebenen Vieraugenprinzips von einem/einer qualifizierten Revisor(in) geprüft wird (vgl. Bredel/Maaß 2016b: 71). Der/Die Revisor(in), der/die ebenfalls die Regeln der Leichten Sprache beherrschen und die in Kapitel 4.3 genannten Kompetenzen haben muss, prüft den Text auf Regelkonvergenz auf morphologischer, syntaktischer, semantischer und textueller Ebene und evaluiert zudem die Funktionalität des Textes in der Zielsituation. Die Korrekturen können dabei je nach Absprache entweder von dem/der Revisor(in) oder dem/ der Übersetzer(in) eingearbeitet werden. Wird der Text als regelkonform und funktional befunden, wird er mit einem Prüfsiegel versehen (vgl. Bredel/Maaß 2016a: 137).

Im Bereich der Barrierefreien Kommunikation gibt es somit verschiedene Arten der Revision, die, sofern dies aus administrativer, personeller und finanzieller Hinsicht praktikabel ist, bestenfalls in Kombination angewandt werden können. Mit einem doppelten Revisionsprozess, in dem der Text nach Revision durch eine(n) professionelle(n) Revisor(in) eine Zielgruppenprüfung durchläuft, könnte somit sowohl die Forderung der Praxisvertreter, die Zielgruppen Leichter Sprache in den Übersetzungsprozess mit einzubeziehen, erfüllt werden als auch die in der DIN ISO 17100 genannten Qualitätsstandards der Revision eingehalten werden. Trotz der genannten Nachteile der ausschließlichen Zielgruppenprüfung bietet die Einbindung der Zielgruppe in den Prüfprozess jedoch auch Vorteile und sollte somit nicht durchgängig durch eine rein professionelle Prüfung ersetzt werden. Angesichts der Tatsache, dass sich Übersetzer(innen) und Zieltextempfänger(innen) im Hinblick auf ihre schriftlichen, sprachlichen und teilweise auch kognitiven Fähigkeiten unterscheiden, fungieren empirische Erkenntnisse, die bspw. aus Studien zur Aufmerksamkeitsspanne oder zum Wortschatz gewonnen und in den Regelwerken berücksichtigt wurden, zwar als Richtwerte; ob diese Richtwerte und Regeln in der Leichte-Sprache-Praxis tatsächlich ,funktionieren', lässt sich jedoch weder von dem/der Übersetzer(in) noch von dem/der professionellen Revisor(in), sondern lediglich von der Zielgruppe beantworten. Darüber hinaus wird mit der Zielgruppenprüfung zugleich das Kriterium des Einholens von Feedback bereits teilweise erfüllt. Hierbei ist allerdings zu berücksichtigen, dass es angesichts der verstärkt auftretenden Forderung nach barrierefreien Texten in Zukunft kaum möglich sein wird, jeden einzelnen Text einer doppelten Revision zu unterziehen. Dies führt wiederum zur Notwendigkeit, vermehrt CAT-Tools in den Übersetzungsprozess miteinzubinden. Dementsprechend ist auch das umgekehrte Szenario denkbar: So schlagen Maaß et al. (2014) bspw. vor, bestimmte Konstruktionen und Segmente bei der ersten Verwendung von einer Prüfgruppe auf Verständlichkeit prüfen zu lassen und diese anschließend zur Wiederverwendung in Translation Memories zu speichern (vgl. Maaß et al. 2014: 81f.). Mit einer derartigen Lösung könnten ebenfalls zum einen die in der ISO 17100 genannten Anforderungen an die Revision erfüllt werden und zum anderen die Vorteile der Einbindung der Zielgruppe in den Übersetzungsprozess genutzt werden.

Die im Anschluss an die Revision folgenden Teilprozesse, d. h. die fachliche Prüfung und das Korrekturlesen, können in der Leichte-Sprache-Übersetzung in Abstimmung mit dem Kunden/der Kundin ebenfalls optional durchgeführt werden, wobei die fachliche Prüfung der inhaltlichen Korrektheit insbesondere bei fachlichen Texten (z. B. aus dem juristisch-administrativen oder medizinischen Bereich) zu empfehlen ist. Ebenso wie bei der interlingualen Übersetzungsdienstleistung ist es im Anschluss die Aufgabe des Projektmanagers/der Projektmanagerin, dem Kunden/der Kundin den Zieltext zuzustellen. 


\subsection{Projektvor- und -nachbereitung}

Die oben beschriebenen Produktionsprozesse werden entsprechend der ISO 17100 in vorbereitende und nachbereitende Schritte eingebettet. Im Rahmen der Projektvorbereitung wird überprüft, ob die für den Übersetzungsauftrag notwendigen Ressourcen zur Verfügung stehen und die notwendige Expertise vorhanden ist. Ersteres kann beispielsweise den Einsatz von Tools oder Qualitätssicherungsmaßnahmen betreffen. Letzteres beinhaltet die Expertise zum Ausräumen und Auflösen von Kommunikationsbarrieren, die bei Fachtexten insbesondere fachlicher und fachsprachlicher Natur sind (vgl. Rink 2020). Die Erfassung des situativen Kontextes der Übersetzung sowie der intendierten Zielgruppe ist für das Angebot und die Vereinbarung zwischen Übersetzer(in) und Auftraggeber(in) wichtig. Auf dieser Basis erfolgt auch eine zielgruppenorientierte Selektion von Informationen aus dem Ausgangstext und eine Übersetzungsstrategie für den Zieltext.

Vor dem Hintergrund, dass sich die Leichte Sprache in einem kontinuierlichen Entwicklungsprozess befindet, ist auch die Projektnachbereitung im Bereich der Barrierefreien Kommunikation von zentraler Bedeutung. Dies bezieht sich sowohl auf das Feedbackmanagement als auch auf die Projektarchivierung. Im Unterschied zum interlingualen Übersetzungsprozess sollte das Feedback dabei nicht nur von dem Kunden/ der Kundin, sondern auch von den Zieltextrezipient(inn)en, d. h. von Vertreter(inne)n der Zielgruppe eingeholt werden, um Rückschlüsse darauf zu erhalten, ob der Text funktionsgerecht ist und einen barrierefreien Zugriff auf Informationen ermöglicht. Die aus den Rückmeldungen der Zieltextrezipient(inn)en gewonnenen Erkenntnisse sollten in die weitere Übersetzungspraxis miteinbezogen werden. So kann es bspw. im Rahmen der Projektnachbereitung erforderlich sein, bereits bestehende Terminologieeinträge im Nachhinein zu korrigieren oder im Translation Memory bestimmte Konstruktionen auf Satzebene als verständlich bzw. unverständlich zu markieren. Auch hier soll eine optimierte Prozessqualität zu einer nachhaltig optimierten Produktqualität führen.

Wie bereits erwähnt, wird es angesichts des stark anwachsenden Textvolumens in Zukunft kaum realisierbar sein, jeden barrierefreien Text vor der Freigabe von einer qualifizierten Prüfgruppe prüfen zu lassen. Da Leichte-Sprache-Übersetzungen in der derzeitigen Praxis häufig auch von Akteur(inn)en ohne übersetzungswissenschaftlichen Hintergrund angefertigt werden, ist allerdings momentan noch nicht davon auszugehen, dass die oben vorgeschlagene Lösung der Einbindung von CAT-Tools flächendeckend umgesetzt wird. Um dennoch gewährleisten zu können, dass der Text seine intendierte Funktion in der Zielsituation erfüllt, ist das Einholen des Feedbacks der Zieltextleser(innen) somit eine valide Möglichkeit. Die Notwendigkeit, die Übersetzungen auf ihre Funktionalität in der Zielsituation bewerten zu lassen, wird auch in der Richtlinie (EU) 2016/2102 des Europäischen Parlaments und des Rates vom 26. Oktober 2016 über den barrierefreien Zugang zu den Websites und mobilen Anwendungen öffentlicher Stellen berücksichtigt. Mit der Richtlinie werden öffentliche Stellen u. a. dazu verpflichtet, einen Feedback-Mechanismus einzurichten, über den die Nutzer(innen) Mängel mitteilen oder zusätzliche Informationen anfordern können.

Auch der intralinguale Übersetzungsprozess endet mit der Archivierung des Projektes, wobei ebenso wie in der interlingualen Übersetzungsdienstleistung sicherzustellen ist, dass die rechtlichen und vertraglichen Pflichten in Bezug auf den Umgang mit den Daten und den Datenschutz eingehalten werden. Im Bereich der Barrierefreien Kommunikation sollte das Resultat des Übersetzungsprozesses somit ein geprüfter, leicht zugänglicher, gut wahrnehmbarer, leicht verständlicher, sprachlich und inhaltlich korrekter sowie funktionaler Zieltext sein. Die Orientierung an der ISO 17100 fungiert dabei, ebenso wie in der interlingualen Übersetzung, als „Qualitätsausweis“ (Bredel/Maaß 2016b: 72), wobei im Bereich der Leichte-Sprache-Übersetzungen bislang nur wenige Übersetzer(innen) über einen derartigen Qualitätsausweis verfügen.

\subsection{Prozessbeteiligte Experten}

Werden alle in Abschnitt 4.1 beschriebenen Teilprozesse umgesetzt, arbeiten die folgenden Prozessbeteiligten an einem Auftrag:

- Übersetzer(innen) und andere Textschaffende

- Revisor(inn)en

- fachliche Prüfer(innen) 
- Leichte-Sprache-Prüfer(innen)

- Projektmanager(innen)

Die Übersetzer(innen) und weiteren in den Übersetzungsprozess eingebundenen Textschaffenden (z. B. Journalist(inn)en, Redakteur(inn)e(n)) müssen gemäß ISO 17100 die folgenden Kompetenzen nachweisen: übersetzerische Kompetenz, sprachliche und textliche Kompetenz, Kompetenz beim Recherchieren, bei der Informationsgewinnung und -verarbeitung, kulturelle Kompetenz, technische Kompetenz und Sachgebietskompetenz. Im Falle der Übersetzung in Leichte und Einfache Sprache müssen darüber hinaus noch die sprachlichen Regeln der entsprechenden Varietät bekannt sein; außerdem muss Wissen im Umgang mit der Zielgruppe und deren kommunikativen Kontexten vorhanden sein. Die am Qualitätssicherungsprozess beteiligten Revisor(inn)en müssen zusätzlich zu diesen für die Übersetzer(innen) genannten Kompetenzen Revisionskompetenz vorweisen. Bei den fachlichen Prüfer(inne)n handelt es sich um Sachgebietsspezialisten, die den Text auf inhaltlich-fachliche Korrektheit prüfen.

Nach dem diskursivierten Expertenbegriff zählen auch die Beteiligten an der Zielgruppenprüfung zu den prozessbeteiligten Expert(inn)en. Hier sind die Prüfer(innen) selbst zu nennen sowie die Moderator(inn)en. Die Moderator(inn)en benötigten die gleichen Kompetenzen wie die Übersetzer(innen). Neben ausgeprägtem Zielgruppenwissen werden auch sogenannte Soft Skills wie Kommunikationsfähigkeit und Einfühlungsvermögen benötigt. Prüfer(innen) zeichnen sich dadurch aus, dass sie der Zielgruppe angehörig sind und auf Wahrnehmungsund Verständlichkeitsprobleme aufmerksam machen, für die Optimierungspotenzial besteht.

Der/Die Projektmanager(in) sollte die Fähigkeiten besitzen, alle Phasen des Übersetzungsprozesses kompetent zu koordinieren und zu begleiten. Dies erfordert zum einen tiefgehende Kenntnisse über die Abfolge und Umsetzung der Teilprozesse und zum anderen tiefgreifendes Wissen über die Übersetzerdienstleistungsbranche für Leichte und Einfache Sprache. Anzumerken ist, dass Projektmanager(in) und Übersetzer(in) durchaus eine Person sein können, was bei der Qualitätssicherung durch das Vieraugenprinzip nicht der Fall sein darf. Hier müssen Übersetzer(in) und Revisor(in) unterschiedliche Beteiligte sein. Je nach Qualitätssicherungsprozess bzw. Grad der fachlichen Prüfung müssen nicht alle hier beschriebenen Experten involviert sein. Die Auswahl der Beteiligten hängt von der Art und vom Umfang der Qualitätssicherungsschritte ab.

\section{Ausblick}

Im Jahr 1984 stellt Holz-Mänttäri die folgende Problematik für die interlinguale Translation dar:

[Hier] kann man wohl von einem Teufelskreis sprechen. Er kann nur im Wissenschaftsbereich wirklich durchbrochen werden, weil nur dort ,Erkenntnis‘ der Bedingungen zu konstruktiven Maßnahmen führen kann. Bohrende Fragen, wie sie zum jetzigen historischen Zeitpunkt im Praxisbereich immer lauter gestellt werden, können den Erkenntnisprozess fördern. Insofern könnte ein gemeinsames Bemühen um die gemeinsame Sache seitens der vier Aspektvertreter

- translatorischer Bedarfsträger

- translatorischer Produzent

- translatologischer Ausbilder

- translatologischer Forscher

zum gegenwärtigen historischen Zeitpunkt von großem Nutzen sein. Die Entwicklung hat wohl die Marge erreicht, wo naturwüchsiges und intuitives Handeln der vier Aspektvertreter den Anforderungen nicht mehr gerecht wird und für das Gesamtgefüge ,Gemeinschaft‘ nicht mehr tragbar ist. (Holz-Mänttäri 1984: 165).

Dieser Teufelskreis gilt für die interlinguale Translation nun seit geraumer Zeit als durchbrochen, da einerseits die praxeologischen Ansätze innerhalb der Forschung nicht mehr als stigmatisierend abgewehrt, sondern befruchtend in die angewandte Translationsforschung eingebettet werden. Andererseits haben sich in enger Kooperation zwischen Berufsverbänden und universitären Ausbildungsstätten praxisrelevante Forschungszweige und berufsbildende Studiengänge entwickelt. Für die interlinguale Übersetzung stellt daher niemand mehr in Frage, dass Professionalisierung über den Weg der Akademisierung erfolgt. 
Die intralinguale Translation in Einfache und Leichte Sprache steht noch am Anfang dieses Professionalisierungswegs. Es gilt, wie von Holz-Mänttäri beschrieben, auch hier die diversen Aspektvertreter zum gemeinsamen Bemühen um die gemeinsame Sache zu motivieren. Dafür müssen allerdings grundlegende Fragen zur Produkt- und Prozessqualität hinterfragt und beforscht werden. Regeln und Textsortenkonventionen müssen empirisch validiert werden. Durch gegenseitige Offenheit kann auch für die intralinguale Translation die Akademisierung auf dem Weg zur Professionalisierung helfen.

Bibliografía:

Ahrens, S. (2020): "Easy Language and Administrative Texts: Second Language Learners as a Target Group", in HansenSchirra S. und MaßßC.(eds.), Easy Language Research: Text and User Perspectives, Frank \& Timme, Berlin, 67-97. https://doi. org/10.26530/20.500.12657/42088

Behindertengleichstellungsgesetz des Bundes (BGG) (2018) $[\mathrm{http}: / /$ www.gesetze-iminternet.de/bgg/index.html].

Bredel, U., und Maaß, C. (eds.) (2016a) Leichte Sprache. Theoretische Grundlagen. Orientierung für die Praxis, Duden, Berlin.

Bredel, U., und Maaß, C. (eds.) (2016b) Ratgeber Leichte Sprache. Die wichtigsten Regeln und Empfehlungen für die Praxis, Duden, Berlin.

Clark, H. (1996): Using Language, Cambridge University Press, New York. https://doi org/10.1017/CBO9780511620539.

Deutscher Bundestag (2015): Entwurf des Gesetzes zur Weiterentwicklung des Behindertengleichstellungsrechts (BGG) [http://www.bmas.de/ SharedDocs/Downloads/DE/PDFMeldungen/2015/referentenentwurf-einesgesetzes-zur-weiterentwicklung-desbehindertengleichstellungsrechts.pdf? blob=publicationFile $\& v=3$ ]

DIN EN ISO 17100 (2015): Übersetzungsdienstleistungen - Anforderungen an Übersetzungsdienstleistungen, Beuth, Berlin.

Hansen-Schirra, S., Bisang, W., Nagels, A. Gutermuth, S., Fuchs, J., Borghardt, L., Deilen, S., Gros, A.-K., Schiffl, L. und Sommer, J. (2020a): "Intralingual Translation into Easy Language - O how to Reduce Cognitive Processing Costs", in Hansen-Schirra S. und Maaß C. (eds.), Easy Language Research: Text and User Perspectives, Frank \& Timme, Berlin, 197-225. https://doi. org/10.26530/20.500.12657/42088

Hansen-Schirra, S., Nitzke, J., Gutermuth, S., Maaß, C., und Rink, I. (2020b): "Technologies for Translation of Specialized Texts into Easy Language", in Hansen-
Schirra S. und MaaßC. (eds.), Easy Language Research: Text and User Perspectives, Frank \& Timme, Berlin, 99-127. https://doi. org/10.26530/20.500.12657/42088

Holz-Mänttäri， J. (1984): Translatorisches Handeln. Theorie und Methode. Suomalainen Tiedeakatemia, Helsinki.

Inclusion Europe (2009): Informationen für alle! Europäische Regeln, wie man Informationen leicht lesbar und leicht verständlich macht. [https://www.lag-abt-niedersachsen.de/ uploads/migrate/Download/Infofralle.pdf].

Jakobson, R. (1959): "On linguistic aspects of translation", in Brower R. (ed.) On Translation, Harvard University Press, Cambridge, 232-239. https://doi org/10.4159/harvard.9780674731615.c18.

Leidner, R. (2007): "Die Begriffe »Barrierefreiheit«, »Zugänglichkeit» und »Nutzbarkeit« im Fokus", in Föhl P. Erdrich S., John H. und Maaß K. (eds.), Das barrierefreie Museum. Theorie und Praxis einer besseren Zugänglichkeit, transcriptVerlag, Bielefeld, 28-33.

Maaß, C. (2015): Leichte Sprache. Das Regelbuch, Lit-Verlag, Münster. https://doi. org/10.25528/018

Maaß, C., Rink, I., und Zehrer, C. (2014): "Leichte Sprache in der Sprach- und Übersetzungswissenschaft", in Jekat S. Jüngst H., Schubert K., und Villiger Claudia (eds.), Sprache barrierefrei gestalten. Perspektiven aus der Angewandten Linguistik, Frank \& Timme, Berlin, 53-86.

Maaß, C. (2019): "Übersetzen in Leichte Sprache“, in Maaß C. und Rink I. (eds.), Handbuch Barrierefreie Kommunikation, Frank \& Timme, Berlin, 273-302. https:// doi.org/10.25528/021

Maaß, C., und Rink, I. (2019): "Über da Handbuch Barrierefreie Kommunikation", in Maaß C. und Rink I. (eds.), Handbuch Barrierefreie Kommunikation, Frank \& Timme, Berlin, 17-25. https://doi. org/10.25528/019.

Maa8. C. (2020): Easy Language - Plain Language - Easy Language Plus. Balancing comprehensibility and acceptability. Frank \& Timme, Berlin. https://doi.org/10.25528/042 Mälzer, N. (2016): "Zur Einführung”, in Mälzer N. (ed.), Barrierefreie Kommunikation Perspektiven aus Theorie und Praxis, Frank \& Timme, Berlin, 7-11.

Netzwerk Leichte Sprache (2013): Die Regeln für Leichte Sprache, [https://www.leichtesprache.org/wp-content/uploads/2017/11/ Regeln_Leichte_Sprache.pdf].

Nord, C. (1993): Einführung in das funktionale Übersetzen. Am Beispiel von Titeln und Überschriften, Francke, Tübingen. https:// doi.org/10.1075/babel.41.1.17wil.

Reiß, K., und Vermeer, H. (eds.) (1984): Grundlegung einer allgemeinen Translationstheorie, Niemeyer, Tübingen. https://doi. org/10.1515/9783111351919

Richtlinie (EU) 2016/2102 des Europäischen Parlaments und des Rates vom 26. Oktober 2016 über den barrierefreien Zugang zu den Websites und mobilen Anwendungen öffentlicher Stellen (2016) [https://eur-lex.europa. eu/legal-content/DE/TXT/PDF/?uri=CEL EX:32016L2102\&from=DE]

Rink, I. (2019): “ Kommunikationsbarrieren“, in Maaß C. und Rink I. (eds.), Handbuch Barrierefreie Kommunikation, Frank \& Timme, Berlin, 29-65. https://doi.org/10.25528/020

Rink, I. (2020): Rechtskommunikation und Barrierefreiheit: Zur Übersetzung juristischer Informations- und Interaktionstexte in Leichte Sprache, Frank \& Timme, Berlin. https://doi.org/10.26530/20.500.12657/432 15

Risku, H. (1998): Translatorische Kompetenz: kognitive Grundlagen des Übersetzens als Expertentätigkeit, Tübingen, Stauffenburg.

Siever, H. (2010): Übersetzen und Interpretation. Die Herausbildung der Übersetzungswissenschaft als eigenständige wissenschaftiche Disziplin im deutschen Sprachraum von 1960 bis 2000, Lang, Frankfurt/M

Wolfer, S. (2017): Verstehen und Verständlich keit juristisch-fachsprachlicher Texte, Narr, Tübingen. 